\title{
Use of Laser Irradiation to Form Anti-Corrosive Surface Oxide Layer on Mg Metal
}

\author{
Shogo Izumi*1, Michiaki Yamasaki*2, Masaaki Otsu and Yoshihito Kawamura \\ Department of Materials Science and Enginnering, Kumamoto University, Kumamoto 860-8555, Japan
}

Laser irradiation was used to improve the poor corrosion resistance of commercial grade pure magnesium (99.9 mass $\%$ ) metal. The magnesium metal plates were immersed in a saturated $\mathrm{Mg}(\mathrm{OH})_{2}$ aqueous solution and the hydroxide layer formed on the plates was decomposed by laser-irradiation in air to form a uniform anti-corrosive oxide layer. Light scanning confocal microscopy (LSCM) was used to measure the thickness of the oxide layer and characterize the surface morphology of the laser-irradiated specimens. Corrosion resistance of the laserirradiated specimens was evaluated by the method of hydrogen evolution during immersion using a $1 \mathrm{mass} \% \mathrm{NaCl}$ aqueous solution. This study revealed that low speed-scanning with a low heat-input laser formed a thick, uniform $\mathrm{MgO}$ layer by decomposition of the $\mathrm{Mg}(\mathrm{OH})_{2}$ formed by primary immersion. The $\mathrm{MgO}$ layer prevented filiform corrosion and improved corrosion resistance of $\mathrm{Mg}$ metal.

[doi:10.2320/matertrans.L-MRP2007859]

(Received February 8, 2007; Accepted April 20, 2007; Published June 20, 2007)

Keywords: Magnesium, $\mathrm{MgO}$, Corrosion, Surface treatment, Laser irradiation

\section{Introduction}

Magnesium alloys have recently attracted a great deal of attention as structural materials because of their lightweight and high specific strength. In general, $\mathrm{Mg}$ alloys produced by traditional ingot metallurgy $(\mathrm{I} / \mathrm{M})$ processing have lower corrosion resistance. The main reason for the low corrosion resistance of $\mathrm{Mg}$ and $\mathrm{Mg}$ alloys is internal galvanic attack due to impurities and/or alloying elements. The application of rapid solidification processing to $\mathrm{Mg}$ alloys results in reduction in grain and intermetallic particle sizes, extended solid solubility, formation of a non-equilibrium phase, and improved chemical homogeneity. Enhancement of chemical homogeneity by rapid solidification of the optimum alloy composition may result in suppression of internal galvanic corrosion. ${ }^{1,2)}$ However, because it is important to prevent contact corrosion as well as inhibiting internal galvanic corrosion, surface treatment is required to form an insulating protective layer on magnesium metal. To improve the surface properties, a variety of surface treatment technologies have been proposed and investigated, including conversion coating, plating, anodizing, fluoride coating, and surface lasermelting. ${ }^{3-6)}$

Recently, Tribollet and Baril et al. ${ }^{7)}$ proposed a kinetic model for magnesium corrosion that takes into account the Negative Difference Effect (NDE). ${ }^{8,9)}$ In their model, the surface of $\mathrm{Mg}$ alloys is coated with an insulating inner film of $\mathrm{MgO}$ with some small gaps in addition to an outer $\mathrm{Mg}(\mathrm{OH})_{2}$ layer. The chemical reaction corresponding to the NDE occurs on the $\mathrm{MgO}$-free areas. Conversely, the $\mathrm{MgO}$ inner layer plays an important role in the corrosion protection of magnesium metal and its alloys. Yamamoto et al. ${ }^{10)}$ proposed a new artificial corrosion-oxidization method to improve corrosion resistance of AZ31 magnesium alloys. They treated the alloys at $673-773 \mathrm{~K}$ for $1 \mathrm{~h}$ in air to create an artificial protective $\mathrm{MgO}$ layer by conversion of magnesium hydrox-

\footnotetext{
${ }^{* 1}$ Graduate Student, Kumamoto University

${ }^{* 2}$ Corresponding author, E-mail: yamasaki@gpo.kumamoto-u.ac.jp
}

ide into magnesium oxide. As mentioned above, this artificial corrosion-oxidization method is one of the surface treatment technologies that have attracted considerable research interest. However, when this method is applied to rapidly solidified and age-hardened $\mathrm{Mg}$ alloys, the high temperature treatment damages microstructures. Therefore, in this study we formed a stable $\mathrm{Mg}(\mathrm{OH})_{2}$ film on $\mathrm{Mg}$ metal by immersion in a saturated $\mathrm{Mg}(\mathrm{OH})_{2}$ solution, and then laser-irradiated the surface of the $\mathrm{Mg}$ metal to convert the hydroxide into a protective oxide layer without grain coarsening in the substructure of the metal. It was anticipated that corrosion resistance would be improved by this laser-irradiation surface modification method. The aim of this experimental study was to ascertain whether laser irradiation enhanced corrosion resistance. Particular attention was paid to variations in the formation of $\mathrm{MgO}$ from $\mathrm{Mg}(\mathrm{OH})_{2}$ on the surface with changes in laser-irradiation condition.

\section{Experimental Procedure}

The specimen material in this study was a commercial grade 99.9 mass\% pure magnesium $(3 \mathrm{~N}-\mathrm{Mg})$, chemical composition of which is shown in Table 1. Specimens of about $10 \times 10 \times 5 \mathrm{~mm}^{3}$ were mechanically polished with silicon carbide lapping paper up to \# 4000 grit. The specimens were immersed in saturated $\operatorname{Mg}(\mathrm{OH})_{2}$ aqueous solution at room temperature for 5, 10 and $15 \mathrm{~min}$, and then laser-irradiated in air. A Q-switched Nd: $\mathrm{YVO}_{4}$ laser (YVO Inc., LT-020 i-marker) with an average power output of around $11 \mathrm{~W}$ and a wavelength of $1064 \mathrm{~nm}$ was used. The Qswitch frequency was $200 \mathrm{kHz}$. The beam focus diameter was approximately $50 \mu \mathrm{m}$, the laser scan spacing was $10 \mu \mathrm{m}$, and the scanning speeds were $25,50,100$, and $200 \mathrm{~mm} \mathrm{~s}^{-1}$.

Table 1 Chemical composition of $3 \mathrm{~N}-\mathrm{Mg}$ (mass\%).

\begin{tabular}{cccccccc}
\hline $\mathrm{Al}$ & $\mathrm{Zn}$ & $\mathrm{Mn}$ & $\mathrm{Si}$ & $\mathrm{Cu}$ & $\mathrm{Ni}$ & $\mathrm{Fe}$ & $\mathrm{Mg}$ \\
\hline 0.0013 & 0.003 & 0.012 & 0.024 & 0.0022 & 0.0003 & 0.0014 & Bal.
\end{tabular}



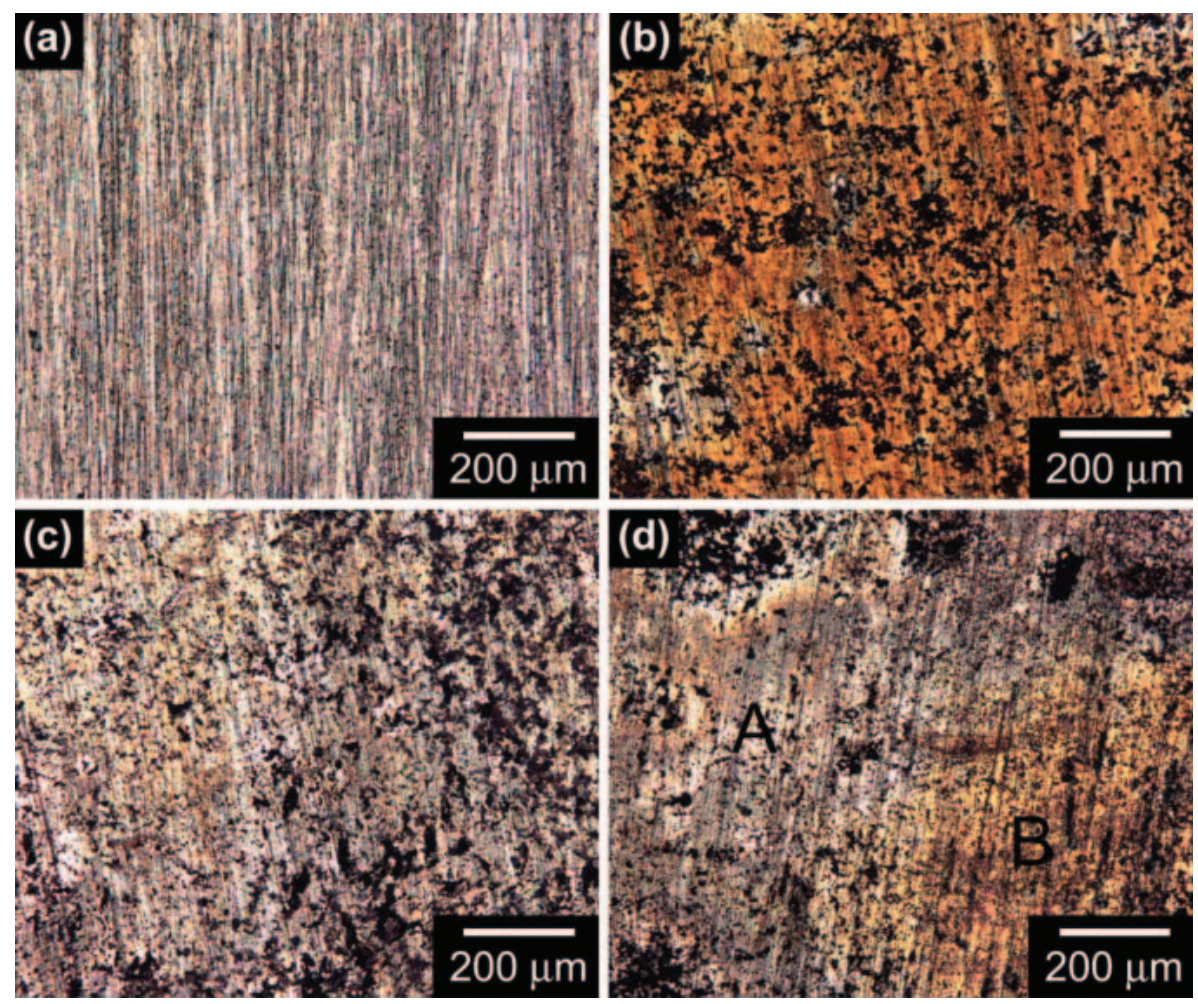

Fig. 1 (color). LSCM image of the surface morphology of various surface-treated $3 \mathrm{~N}-\mathrm{Mg}$ specimens. (a) As-mechanically polished, (b) as-immersed in a saturated $\mathrm{Mg}(\mathrm{OH})_{2}$ aqueous solution for $10 \mathrm{~min}$, laser-irradiated at a scanning speed of (c) $50 \mathrm{~mm} \mathrm{~s} \mathrm{~s}^{-1}$ and (d) $200 \mathrm{~mm} \mathrm{~s}^{-1}$

The structure of the laser-irradiated specimens was investigated by the grazing incidence X-ray diffractometry (XRD) using $\mathrm{Cu} K \alpha$ radiation at the incident angle of $1^{\circ}$. A Lasertec model C-130 real color light scanning confocal microscope (LSCM) was used to measure the film thickness and characterize the surface morphology of each of the surface-treated alloys.

The corrosion tests were carried out in 1.0 mass $\% \mathrm{NaCl}$ aqueous solution at $298 \pm 0.1 \mathrm{~K}$. The dissolution rates were determined by hydrogen evolution during immersion. ${ }^{1)}$ The corrosion rate, $R_{c}\left(\mathrm{~mm} \mathrm{year}{ }^{-1}\right)$, was calculated using the following formula;

$$
R_{c}=\frac{21202 \cdot \Delta V_{h} \cdot M}{\rho \cdot A \cdot t},
$$

where $V_{h}$ is the volume of hydrogen evolved in $\mathrm{ml}, M$ is the apparent atomic weight of the specimen in $\mathrm{g} \mathrm{mol}^{-1}, \rho$ is the density of the specimen in $\mathrm{g} \mathrm{cm}^{-3}, A$ is the surface area of the specimen in $\mathrm{mm}^{2}$, and $t$ the test duration in minutes. Specimens were immersed for $3000 \mathrm{~min}$. The corrosion rate was averaged from five tests.

\section{Results and Discussion}

Figure 1 shows LSCM images of the surface morphology of the $3 \mathrm{~N}-\mathrm{Mg}$ specimen after mechanical polishing (a), after primary immersion in saturated $\mathrm{Mg}(\mathrm{OH})_{2}$ solution for $10 \mathrm{~min}$ (b), and after laser irradiation (c, d). After primary immersion, the color of the surface changed to yellow. Furthermore, black convex-shaped precipitates formed on the surface during immersion. The surface of the specimen laser- irradiated at scanning speeds of 50 and $200 \mathrm{~mm} \mathrm{~s}^{-1}$ after primary immersion are shown in Figs. 1(c) and (d), respectively. Light brown (A) and yellow (B) regions were observed on the surface of the specimen laser-irradiated at a scanning speed of $200 \mathrm{~mm} \mathrm{~s}^{-1}$, although there was no yellow region in the specimen laser-irradiated at a scanning speed of $50 \mathrm{~mm} \mathrm{~s}^{-1}$. The yellow (B) region may correspond to an area where $\operatorname{Mg}(\mathrm{OH})_{2}$ remains. In this study, the laser output was set to $11 \mathrm{~W}$, which was the maximum output before a melting mark appeared on the surface. Total heatinput was controlled by changing the laser scanning speed. High total heat-input (low scanning speed) caused surface modification such as color change from yellow to light brown. LSCM was used in the line-scanning mode to measure a cross-section profile through the surface layer. From ray-reflection first principles, ray 1 was reflected from the air/surface layer interface, while ray 2 was reflected from the surface layer/metal substrate interface, as illustrated in Fig. 2(a). For normal incident condition and assuming no light absorption in the surface layers, the reflected intensity of ray 1 and 2 can be expressed using Fresnel's equations as follows:

$$
\begin{aligned}
& I_{1}=I_{0}\left(\frac{n_{l}-n_{0}}{n_{l}+n_{0}}\right)^{2}, \\
& I_{2}=I_{0}\left(\frac{4 n_{l} \cdot n_{0}}{\left(n_{l}+n_{0}\right)^{2}}\right)^{2}\left(\frac{n_{l}-n_{m}}{n_{l}+n_{m}}\right)^{2} .
\end{aligned}
$$

Here, $I_{0}$ is the incident intensity and $n_{0}, n_{l}$, and $n_{m}$ are the indices of refraction for air, surface layers, and the metal substrate. Figures $2(\mathrm{~b}, \mathrm{c})$ show typical $\mathrm{x}-\mathrm{z}$ scanning image and the cross-section profile of the specimen, respectively. 


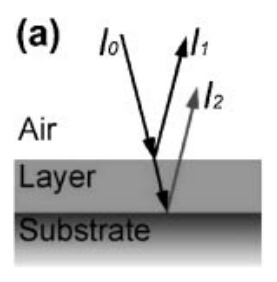

(b)
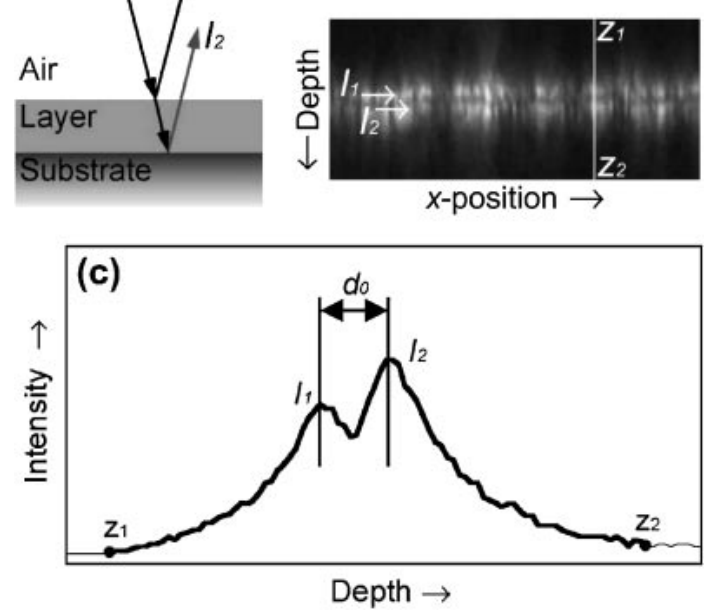

Fig. 2 (a) Schematic diagram of the three-medium (ambient-film-substrate) model. (b) Typical x-z scanning image and (c) cross-section profile of a specimen immersed in a saturated $\mathrm{Mg}(\mathrm{OH})_{2}$ aqueous solution for $10 \mathrm{~min}$.

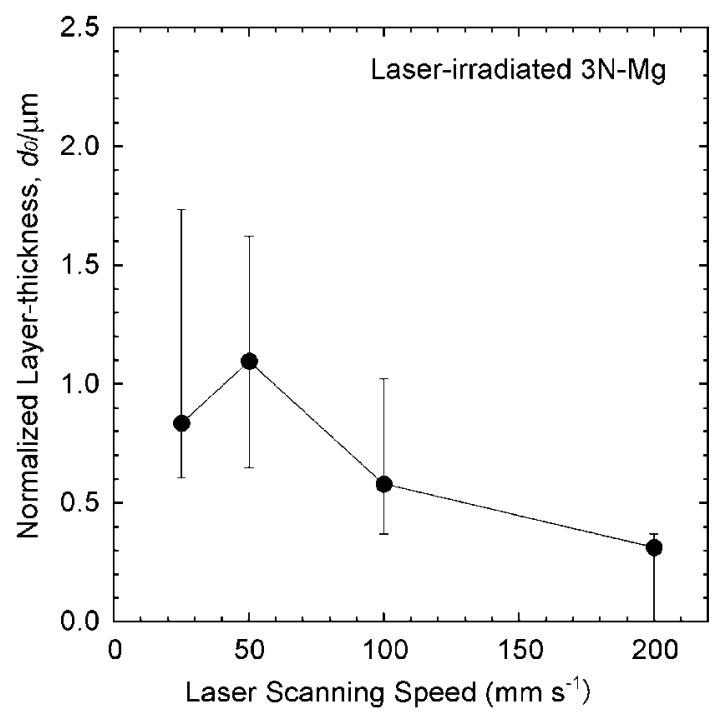

Fig. 3 Change in the normalized layer-thickness, $d_{0}$, as a function of the laser-scanning speed. Prior to laser irradiation, specimens were immersed in a saturated $\operatorname{Mg}(\mathrm{OH})_{2}$ aqueous solution for $10 \mathrm{~min}$. The error bar represents maximum and minimum thickness measured from same specimen.

The distance between the two interfaces, $d_{0}$, was measured by LSCM. Assuming travel through a medium with a refractive index of 1 , the layer thickness, $d_{l}$, can be expressed using following equation:

$$
d_{l}=n_{l} \cdot d_{0} .
$$

Figure 3 shows the change in the normalized layer-thickness, $d_{0}$, as a function of the laser scanning speed. The maximum value of the normalized layer-thickness occurs at a laser scanning speed of $50 \mathrm{~mm} \mathrm{~s}^{-1}$. The normalized layer-thickness decreases with increasing laser-scanning speed. For the specimen laser-irradiated at a scanning speed of $200 \mathrm{~mm} \mathrm{~s}^{-1}$, it was difficult to clearly detect two intensity peaks on the LSCM cross-section profile because the layer thickness was

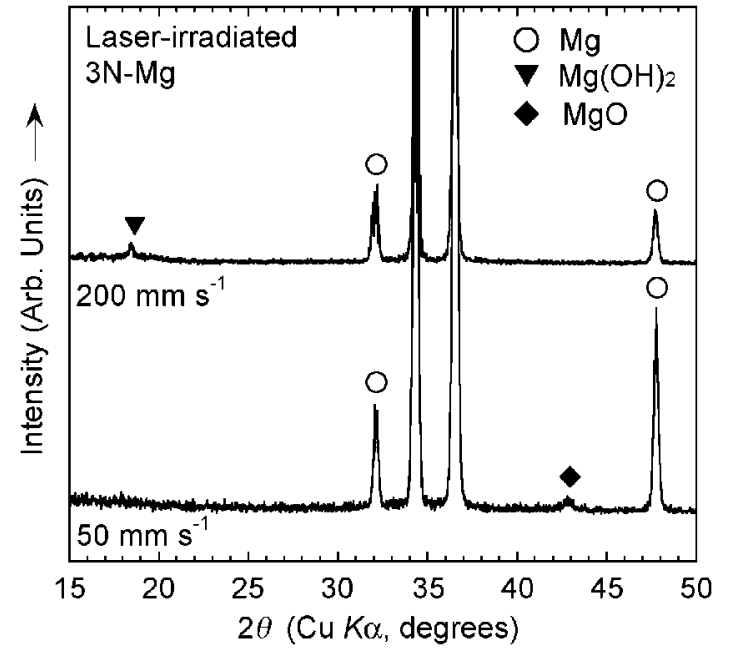

Fig. 4 Grazing incidence XRD patterns of the laser-irradiated specimens with laser-scanning speeds of 50 and $200 \mathrm{~mm} \mathrm{~s}^{-1}$. The incident angle was $1^{\circ}$.

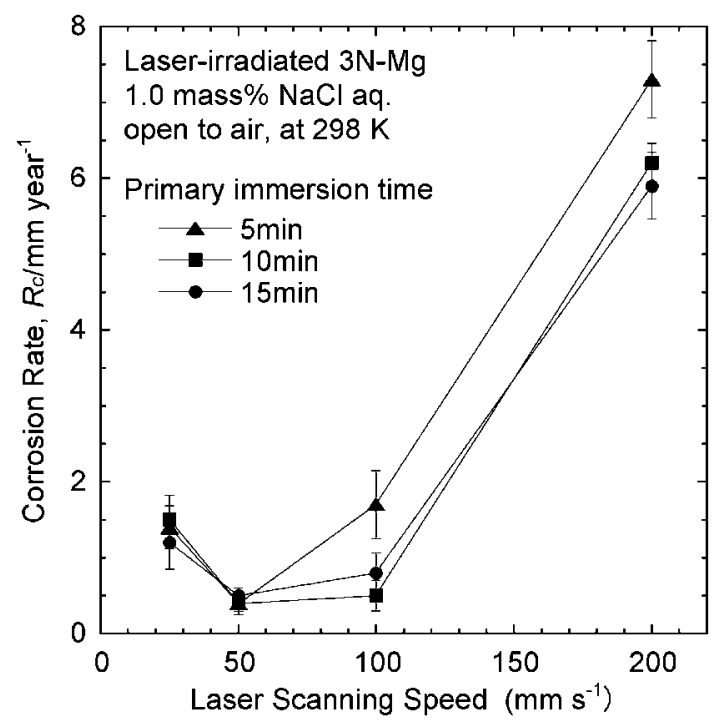

Fig. 5 Change in the initial corrosion rate of the laser-irradiated $3 \mathrm{~N}-\mathrm{Mg}$ in 1.0 mass $\% \mathrm{NaCl}$ aqueous solution as a function of the laser-scanning speed. The error bar represents the standard error.

not homogeneous and was too thin in places. Figure 4 shows the XRD patterns of the laser-irradiated specimens. The $\mathrm{MgO}$ peaks were detected at laser-scanning speeds of 25, 50, and $100 \mathrm{~mm} \mathrm{~s}^{-1}$, although $\mathrm{MgO}$ peaks were not detected in the specimen laser-irradiated at a scanning speed of $200 \mathrm{~mm} \mathrm{~s}^{-1}$. Assuming the refractive index of $\mathrm{MgO}$ is approximately $1.64 \sim 1.74$ at various wavelength, the $\mathrm{MgO}$ layer formed on the surface of the laser-irradiated specimen using a laserscanning speed of $50 \mathrm{~mm} \mathrm{~s}^{-1}$ was calculated to be about $1.74 \sim 1.91 \pm 0.63 \mu \mathrm{m}$ using eq. (4).

Figure 5 shows the changes in the corrosion rate of laserirradiated $\mathrm{Mg}$ metals in 1.0 mass $\% \mathrm{NaCl}$ aqueous solution as a function of the laser scanning speed. The primary immersion treatment times were 5, 10 and $15 \mathrm{~min}$. Primary immersion of greater than $10 \mathrm{~min}$ was a required for the formation of a homogeneous $\mathrm{Mg}(\mathrm{OH})_{2}$ layer, and specimens laser-irradiated at scanning speeds of 50 and $100 \mathrm{~mm} \mathrm{~s}^{-1}$ had 

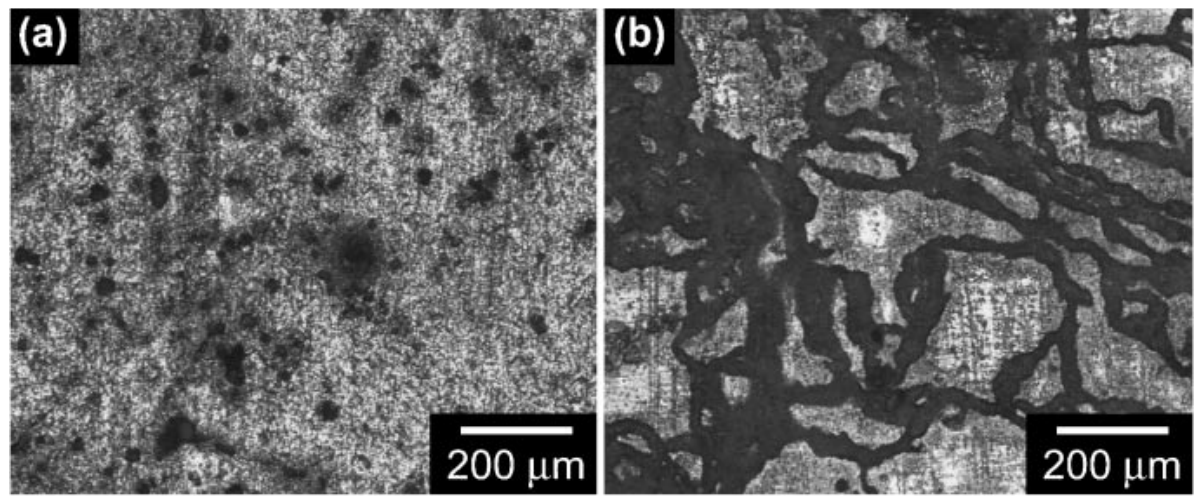

Fig. 6 LSCM images of the corroded surface of a specimen laser-irradiated at a scanning speed of (a) $50 \mathrm{~mm} \mathrm{~s}^{-1}$ and (b) $200 \mathrm{~mm} \mathrm{~s}^{-1}$.

high corrosion resistance although the non-treated (aspolished) and immersion-treated specimens showed high corrosion rate of about 8.8 and $4.8 \mathrm{~mm} \mathrm{year}^{-1}$, respectively. Furthermore, the specimen laser-irradiated at a scanning speed of $50 \mathrm{~mm} \mathrm{~s}^{-1}$ showed high corrosion resistance regardless of differences in immersion time. Figure 6 shows LSCM images of the corroded surface of laser-irradiated specimens. A metallic luster was observed over the whole surface of the specimen laser-irradiated at a scanning speed of $50 \mathrm{~mm} \mathrm{~s}^{-1}$. In contrast, filiform corrosion was observed on the surface of the specimen laser-irradiated at a scanning speed of $200 \mathrm{~mm} \mathrm{~s}^{-1}$, as shown in Fig. 6(b). The corrosion behavior of the laser-irradiated $\mathrm{Mg}$ metals is consistent with the formation of a $\mathrm{MgO}$ layer. The thick $\mathrm{MgO}$ layer formed by laser irradiation inhibits filiform corrosion.

In previous research, it has been reported that the laser melting technique is effective for improving the corrosion resistance of $\mathrm{Mg}$ alloys. ${ }^{4-6)}$ High-heat input of more than $400 \mathrm{~W}$ brings about melting and then rapid quenching in the surface region and substructure near the surface during laserirradiation. They concluded that the rapidly quenched microstructure has high corrosion resistance. In contrast, with low heat input laser irradiation of only $11 \mathrm{~W}$, surface modification from $\mathrm{Mg}(\mathrm{OH})_{2}$ to $\mathrm{MgO}$ occurs without melting of the substrate alloy. Low heat input laser irradiation is a suitable surface treatment method for rapidly solidified and agehardened $\mathrm{Mg}$ alloys, because weak laser irradiation probably has no influence on the substructure of the alloys.

\section{Summary}

A new technique is proposed for improving the poor corrosion resistance of magnesium. Low-speed scanning with a low heat-input laser formed a thick, uniform $\mathrm{MgO}$ layer by decomposition of the $\mathrm{Mg}(\mathrm{OH})_{2}$ layer formed by primary immersion. The optimum conditions for laser irradiation in this study were $11 \mathrm{~W}$ of heat input and a 50 $\mathrm{mm} \mathrm{s}^{-1}$ laser-scanning speed. The thick, uniform $\mathrm{MgO}$ layer on the $\mathrm{Mg}$ metal enhanced corrosion resistance by preventing filiform corrosion.

\section{Acknowledgements}

This work is financially supported by the grant-in-aid for Scientific Research A (No. 19206075) from Ministry of Education, Culture, Sports, Science, and Technology, Japan.

\section{REFERENCES}

1) M. Yamasaki, N. Hayashi, S. Izumu and Y. Kawamura: Corros. Sci. 49 (2007) 255-262.

2) M. Yamasaki, K. Nyu and Y. Kawamura: Mater. Sci. Forum 419-422 (2003) 937-942.

3) J. E. Gray and B. Luan: J. Alloys Compd. 336 (2002) 88-113.

4) D. Dube, M. Fiset, A. Couture and I. Nakatsugawa: Mater. Sci. Eng., A 299 (2001) 38-45.

5) C. Padmavathi, J. K. Sarin Sundar, S. V. Joshi and K. Prasad Rao: Mater. Sci. Technol. 22 (2006) 583-589.

6) S. Y. Liu, J. D. Hu, Y. Yang, Z. X. Guo and H. Y. Wang: Appl. Surf. Sci. 252 (2005) 1723-1731.

7) G. Baril, G. Galicia, C. Deslouis, N. Pebere, B. Tribollet and V. Vivier: J. Electrochem. Soc. 154 (2007) C108-113.

8) G. Baril, C. Blanc and N. Pebere: J. Electrochem. Soc. 148 (2001) B489.

9) G. Song and A. Atrens: Adv. Eng. Mater. 5 (2003) 837.

10) A. Yamamoto and H. Tsubakino: Mater. Trans. 44 (2003) 511-517. 